\title{
Philosophiques
}

\section{Pauline Clochec. Pour lire L'essence du christianisme de Ludwig Feuerbach, Paris, Éditions sociales, 2018, 191 pages}

\section{Emmanuel Chaput}

Volume 46, numéro 1, printemps 2019

URI : https://id.erudit.org/iderudit/1062021ar

DOI : https://doi.org/10.7202/1062021ar

Aller au sommaire du numéro

Éditeur(s)

Société de philosophie du Québec

ISSN

0316-2923 (imprimé)

1492-1391 (numérique)

Découvrir la revue

Citer ce compte rendu

Chaput, E. (2019). Compte rendu de [Pauline Clochec. Pour lire L'essence du christianisme de Ludwig Feuerbach, Paris, Éditions sociales, 2018, 191 pages]. Philosophiques, 46(1), 243-247. https://doi.org/10.7202/1062021ar d'utilisation que vous pouvez consulter en ligne.

https://apropos.erudit.org/fr/usagers/politique-dutilisation/ 


\title{
Comptes rendus
}

\author{
Pauline Clochec. Pour lire L'essence du christianisme de Ludwig \\ Feuerbach, Paris, Éditions sociales, 2018, 191 pages.
}

L'ouvrage de Pauline Clochec, Pour lire L'essence du christianisme de Ludwig Feuerbach, constitue une belle introduction non seulement au magnum opus du philosophe aujourd'hui plus connu pour avoir été l'objet de thèses de Marx que pour son œuvre propre. Il constitue en outre une introduction au contexte historique, philosophique et politique qui mena à la rédaction de l'ouvrage L'essence $d u$ christianisme (I84I), ouvrage qui marqua véritablement son époque.

Le premier mérite de l'auteure est ainsi de chercher à extraire la pensée feuerbachienne du carcan interprétatif hérité du marxisme qui le réduit trop souvent à un simple précurseur avec toute l'ambivalence qu'un tel statut implique:

[L]'épaisseur philosophique de Feuerbach s'en trouve téléologiquement réduite à un "déjà » et un "encore », à ce qui annonce déjà Marx et à ce qui n'est pas encore Marx. Il convient de se prémunir de cette approche marxiste qui a dominé jusqu'à il y a quelques décennies comme d'un obstacle à la compréhension de Feuerbach (p. 17).

Et même si l'auteure retombe elle-même en un sens, à la toute fin de l'ouvrage, dans le piège consistant à évaluer la pensée feuerbachienne à l'aune de sa critique marxienne (p. I67-I68), l'essentiel de l'ouvrage parvient adéquatement à éviter ce travers (à la p. 72 par exemple). Il offre ainsi une excellente porte d'entrée, pour le lecteur francophone, à la pensée feuerbachienne telle qu'elle se présente dans L'essence du christianisme.

L'ouvrage de Clochec ne vise pas en effet à donner une vue générale de la philosophie feuerbachienne. L'auteure se limite de facto aux positions défendues dans L'essence $d u$ christianisme et aux textes ayant contribué de près ou de loin à sa genèse. Il sera ainsi peu ou pas question des orientations subséquentes que Feuerbach donnera à sa pensée. Cela est tout à fait naturel dans la mesure où l'exposition des positions défendues dans L'essence $d u$ christianisme et leur genèse constitue déjà une vaste tâche. Il est cependant plus surprenant de constater l'absence de commentaires sur la réception qu'obtint l'ouvrage à la suite de sa publication, que ce soit dans les milieux conservateurs, catholiques ou protestants, ou chez les Jeunes Hégéliens euxmêmes, comme Bruno Bauer ou Stirner. Les réponses de Feuerbach à ses détracteurs peuvent en effet, par les explications qu'il livre, enrichir notre compréhension de son ouvrage. Cette absence reste néanmoins excusable en raison de l'ampleur des polémiques qu'il aurait fallu traiter. 
L'ouvrage de Clochec se divise essentiellement en trois parties suivant un «triple fil directeur, politique, anthropologique et philosophique» (p. I3). La première retrace le cheminement intellectuel et philosophique de Feuerbach jusqu'à la publication de L'essence du christianisme. La seconde partie présente le cadre historique, philosophique et politique, ainsi que les principales thèses défendues dans l'ouvrage. La troisième partie enfin prend la forme d'une "lecture cursive» (p. I3), c'est-à-dire d'un commentaire rapide de l'ouvrage section par section. Le tout est suivi de courts extraits de L'essence du christianisme traduits par l'auteure ${ }^{1}$.

Les deux premières parties de l'ouvrage sont particulièrement captivantes et permettent de se familiariser avec des textes de jeunesse de Feuerbach souvent peu commentés, mais essentiels pour comprendre son développement au sein de l'École hégélienne, puis sa prise de distance avec celle-ci. L'auteure y montre sa grande maîtrise du contexte historique et politique de l'époque du Vormärz $z^{2}$, ainsi que des débats philosophiques qui marquent l'École hégélienne à l'époque.

Au-delà de cette dimension contextualiste des plus enrichissantes, Clochec démontre également une bonne maîtrise des thèses centrales de L'essence $d u$ christianisme qu'elle parvient à exposer avec clarté. À titre d'exemple, citons ce passage qui résume la conception feuerbachienne de la religion:

La religion est un sentiment subjectif de soi par le détour d'un être imaginaire (Dieu). Elle est donc illusoire, du fait de cette projection de soi hors de soi, mais a un contenu vrai en ce que Dieu n'est que la projection des propriétés humaines $[\ldots]$ Les prédicats divins ne sont donc pas contingents ni ne sont des superstitions arbitraires (p. 88).

Une telle posture, comme le note bien l'auteure, ne saurait être réduite ni à la critique de la religion opérée par les Lumières françaises (p. 57) ni à une tentative de sauvetage du noyau rationnel de la religion à la manière des Lumières kantiennes (p. 88).

Pour Clochec, l'entreprise feuerbachienne consiste essentiellement à extraire du christianisme son noyau anthropologique. Or l'anthropologie feuerbachienne a une dimension fondamentalement sociale, fondée sur la

1. Si on ne peut qu'applaudir la qualité de ces traductions, il est cependant dommage, d'une part, que les références des extraits traduits de L'essence du christianisme soient tirées de l'édition allemande Reclam, alors que l'ensemble des références à L'essence faites dans le commentaire de Clochec est plutôt tiré de l'édition des Gesammelte Werke parue aux éditions Akademie Verlag. Un tableau des équivalences aurait été le bienvenu afin de pouvoir resituer les extraits traduits par rapport au commentaire de l'auteur, et vice versa. Il est aussi dommage d'autre part, surtout pour un ouvrage introductif, de ne pas avoir indiqué les références à la traduction de J.-P. Osier pour le lecteur francophone.

2. "Signifiant littéralement "avant-mars" ", comme l'écrit l'auteure, "ce terme renvoie à la période allant du Congrès de Vienne, en I 8 I 5 , aux révolutions européennes de I 848, celle de l'espace germanique débutant par une insurrection ayant lieu en mars à Berlin» (p. I7). 
relation du Je et du Tu. Cette posture intersubjectiviste que Feuerbach développe dans le cadre de son anthropologie sociale a, comme le souligne l'auteure, une dimension à la fois politique et sociale (p. Io). Elle remet en question non seulement la transcendance divine, mais aussi celle du monarque. Et, plus fondamentalement, elle remet en question l'isolement de l'individu qui fonde l'anthropologie libérale. Cependant, pour Clochec, «si l'anthropologie sociale de Feuerbach s'oppose à une anthropologie individualiste libérale à la Humboldt en faisant de l'être humain un être constitué intersubjectivement, elle partage cependant avec une telle anthropologie libérale la théorie de l'histoire faisant de l'isolement des individus égoïstes le commencement de l'histoire humaine» (p. I67-I68). Ce n'est que progressivement, par le biais d'une éducation (Bildung) historique que l'être humain, à l'instar de l'enfant, en vient à saisir ses rapports de dépendance par rapport à autrui, son appartenance au genre humain, lui qui se conçoit d'abord, du point de vue de la conscience, comme un être isolé et autonome. Telle est en un sens, la thèse interprétative adoptée par Clochec, et c'est ce qui explique à ses yeux le rapport initialement ambivalent de l'individu par rapport à son être générique, et justifie l'émergence de l'illusion théologique:

La saisie primitive de la différence entre "l'individualité", ou "l'existence" empirique, et le "genre", ou "l'essence", rend cependant inévitable en un premier temps la distinction de ce penser infini vis-à-vis de soi-même, et son attribution extra-humaine, limitant l'être humain à son individualité finie. La religion trouve ainsi sa nécessité dans le décalage entre l'existence et l'essence du genre humain (p. 74).

Les qualités indéniables du travail de l'auteure, tant en matière de contextualisation, de vulgarisation que d'argumentation, n'excluent pas cependant que certaines de ses interprétations puissent être soumises au débat. On pense notamment à la distinction faite par Feuerbach entre religion et théologie. Si l'auteure note bien cette distinction (aux pages 44 et 89 notamment), elle semble exclure toute tentative de la part de Feuerbach d'opérer un sauvetage du concept de religion, même sous la forme sécularisée d'une religion de l'humanité: "la théologie est présentée [...] comme un développement nécessaire de la religion, ce qui exclut toute démarche consistant à vouloir sauver la religion contre la théologie» (p. 44). Et pourtant, Feuerbach n'écrira-t-il pas que la philosophie de l'avenir "prend la place de la religion; elle a en elle l'essence de la religion, elle est en vérité elle-même religion ${ }^{3}$ ». Il faut ainsi noter l'usage potentiellement pluriel du concept de religion chez Feuerbach et faire droit à ses tentatives de se réap-

3. Ludwig Feuerbach, «Grundsätze der Philosophie der Zukunft» dans Gesammelte Werke, IX, Berlin, Akademie Verlag, I970, s.340/ Manifestes philosophiques, trad. L. Althusser, Paris, PUF, Io/I8, I973, p. 263. 
proprier l'idée de religion en un sens positif, voire émancipateur ${ }^{4}$. En ce sens, l'auteure nous semble trop catégorique dans son jugement.

Le point de démarcation que l'auteure cherche à établir entre l'approche de Feuerbach et celle d'un Bruno Bauer demanderait lui aussi plus de nuances. Après avoir affirmé que l'approche feuerbachienne «se distingue [...] de celle de Bruno Bauer»(p. 82), Clochec affirme en effet: "Chez Feuerbach, la description de la croyance religieuse se fait ainsi beaucoup moins en termes phénoménologiques de conscience et d'expérience de celleci » (p. 82-83; et dans le même sens, p. I30). Mais une telle affirmation est d'autant plus affaiblie qu'elle affirme par ailleurs que

la méthode de présentation de L'essence du christianisme s'apparente à celle de la Phénoménologie de l'esprit de Hegel. Il s'agit pour Feuerbach, à la suite de Hegel, de décrire le progrès de la conscience même, tout en explicitant des contradictions qui, si elles ne sont pas toujours explicites pour la conscience religieuse, si elles agissent, comme l'écrit Feuerbach en paraphrasant justement Hegel, "dans son dos", n’en déterminent pas moins son évolution (p. I09).

L'auteure se trouve ainsi contrainte à nuancer son propos sans toutefois renoncer à sa thèse: "Quoique, à l'intérieur du mouvement jeunehégélien, Feuerbach se sépare nettement de Bruno Bauer par son naturalisme et son sensualisme, c'est ici le principe central de Bauer - la conscience de soi - qu'il reprend à son compte. La religion est une conscience de soi mystifiée que la philosophie athée doit clarifier»(p. I04). La preuve d'un tel emprunt n'est malheureusement pas démontrée et l'on se demande si L'essence $d u$ christianisme ne revêt pas une dimension phénoménologique plus importante que ne le laisse entendre l'auteure, sans qu'une telle dimension soit pour autant identique ou même redevable à l'approche bauerienne ${ }^{5}$.

Enfin, si Clochec désamorce à bon droit nombre de préjugés hérités du marxisme (selon lesquels Feuerbach ne penserait pas la société ou le travail et serait essentiellement un penseur contemplatif, affirmant l'immédiateté de la nature physique, etc.), elle ne se prononce pas directement sur ce qui demeure l'une des critiques les plus courantes en dehors du marxisme. Nombres de critiques en effet, depuis Max Stirner ${ }^{6}$ jusqu'à Michel Henry ${ }^{7}$, affirment d'une manière ou d'une autre que Feuerbach, loin de démontrer

4. Sur ces usages, voir notre contribution " Emanzipatorische Religiosität“ Friedrich (und Ludwig) Feuerbachs Religion der Zukunft als Kritik am christlichen Individualismus » dans S. Schlüter, T. Polcik, J. Thumann (Hrsg.), Philosophie und Pädagogik der Zukunft. Ludwig und Friedrich Feuerbach im Dialog, Münster, Waxmann, 201 8, s.2II-226.

5. Pour une interprétation de L'essence du christianisme comme phénoménologie de la conscience religieuse, voir le bel ouvrage de Marx W. Wartofsky, Feuerbach, Cambridge, Cambridge University Press, 1982.

6. Cf. Max Stirner, L'Unique et sa propriété et autres écrits, Lausanne, L'Âge d'Homme, I994, $437 \mathrm{p}$.

7. Cf. Michel Henry, «La critique de la religion et le concept de genre dans l'essence du christianisme ", Revue internationale de philosophie, vol. 26, nº IOI, I972, p. 386-404. 
que l'anthropologie constitue le secret de la religion, ne réussit qu'à anthropologiser le divin sous la forme de cette nouvelle idole que serait l'être générique. Que ce soit parce que l'être générique constitue tout autant que Dieu un spectre dominant l'individu (Stirner) ${ }^{8}$ ou simplement parce qu'il ne constitue qu'une version anthropologisée de l'esprit hégélien qui nie le singulier au nom de l'universel (Henry), ces critiques considèrent que Feuerbach ne fait que réhabiliter d'une main ce qu'il critique de l'autre. Certes, l'explicitation de la structure intersubjectiviste de l'anthropologie feuerbachienne peut fournir des éléments de réponses face à ces critiques, mais Pauline Clochec reste assez discrète quant aux rapports qu'entretient l'individu avec l'être générique et sur la place qu'accorde Feuerbach à l'individualité comme telle. Or c'est là, il nous semble, une question qu'il est d'autant plus important d'aborder qu'une bonne partie de l'actualité ou de l'inactualité de la posture feuerbachienne semble en dépendre.

Néanmoins, contre les tentatives de minimiser l'importance de l'entreprise feuerbachienne en soutenant qu'il n'aurait que sécularisé le contenu de la religion en en reproduisant la structure oppressive même, Clochec a raison d'écrire:

La sécularisation, politiquement comme spirituellement, n'est $[\ldots]$ pas une simple laïcisation formelle, laissant intacts les contenus du christianisme et leur enlevant seulement la référence à un Dieu transcendant. C'est l'objet même du christianisme et les normes politiques et morales qu'il justifiait qui sont transformés par le tournant historique auquel Feuerbach, par son livre, entend contribuer (p. I6r).

Pour lire L'essence du christianisme de Ludwig Feuerbach constitue ainsi une excellente introduction à l'ouvrage du philosophe et au contexte au sein duquel il s'inscrit. Les remarques critiques que nous avons pu formuler n'entament en rien ni sa qualité ni son sérieux. Elles montrent au contraire, en un sens, que Pauline Clochec remplit son pari de présenter la pensée feuerbachienne pour et par elle-même en s'extrayant des interprétations de seconde main. Car toute entreprise exégétique, surtout lorsqu'il s'agit d'un auteur jusqu'alors peu discuté, suscite inéluctablement son lot de débats, l'émergence de points de vue divergents, etc. Et il faut, en un sens, plutôt y voir un mérite qu'une lacune que de susciter de tels questionnements qui permettent d'approfondir encore davantage notre compréhension d'une pensée riche et dense.

EMMANUEL CHAPUT

Université d'Ottawa

8. Stirner traitera Feuerbach de "pieux athée» (Stirner, L'Unique et sa propriété et autres écrits, p. 234) en l'accusant de faire de l'Homme, c.-à-d. de l'être générique, le nouveau dieu auquel chacun se doit de correspondre, auquel chacun doit se soumettre. 